\title{
Retrieving aerosol height from the oxygen A band: a fast forward operator and sensitivity study concerning spectral resolution, instrumental noise, and surface inhomogeneity
}

\author{
A. Hollstein and J. Fischer \\ Freie Universität Berlin, Department of Earth Sciences, Institute for Space Sciences, Berlin, Germany \\ Correspondence to: A. Hollstein (andre.hollstein@fu-berlin.de)
}

Received: 6 November 2013 - Published in Atmos. Meas. Tech. Discuss.: 5 December 2013

Revised: 2 April 2014 - Accepted: 4 April 2014 - Published: 22 May 2014

\begin{abstract}
Hyperspectral radiance measurements in the oxygen A band are sensitive to the vertical distribution of atmospheric scatterers, which in principle allows the retrieval of aerosol height from future instruments like TROPOMI, OCO2, FLEX, and CarbonSat. Discussed in this paper is a fast and flexible forward operator for the simulation of hyperspectral radiances in the oxygen A band and, based on this scheme, a sensitivity study about the inversion quality of aerosol optical thickness, aerosol mean height, and aerosol type. The forward operator is based on a lookup table with efficient data compression based on principal component analysis. Linear interpolation and computation of partial derivatives is performed in the much smaller space of expansion coefficients rather than wavelength. Thus, this approach is computationally fast and, at the same time, memory efficient. The sensitivity study explores the impact of instrument design on the retrieval of aerosol optical thickness and aerosol height. Considered are signal to noise ratio, spectral resolution, and spectral sampling. Also taken into account are surface inhomogeneities and variations of the aerosol type.
\end{abstract}

\section{Introduction}

Multiple fields of research can benefit from an accurate and reliable aerosol height product. Among others are atmospheric sciences, where aerosol vertical distribution and interaction with clouds and radiation is discussed (e.g., Chin et al., 2009; Lohmann and Feichter, 2005) as well as long-range aerosol transport (e.g., Betzer et al., 1988; Andreae, 1983) and source attribution (e.g., McConnell et al., 2007; Clarke and Noone, 1985), human health (e.g., Nel, 2005; Harrison and Yin, 2000; Seaton et al., 1995) and pollution studies (McMichael et al., 2003; Pöschl, 2005), and in remote sensing of the atmosphere, where its effect on the retrieval of total aerosol optical thickness is discussed (e.g., Quijano et al., 2000; Duforêt et al., 2007; McClain, 2009; Muller et al., 2007).

Lidar measurements (e.g., using EARLINET; Amodeo et al., 2007), airborne experiments (e.g., Zieger et al., 2007), or balloon ascents (e.g., Rosen et al., 1975) can be used to derive aerosol vertical profiles on local scales, while the backscatter lidar CALIOP on board the satellite CALIPSO (Winker et al., 2009) is currently the only instrument that provides information on a global scale. A limitation of CALIPSO measurements is their sparse spacial and temporal resolution (Winker et al., 2010; Amiridis et al., 2013), which could be improved drastically by deriving aerosol height directly from passive imaging instruments.

Attempts to use the oxygen absorption bands for the retrieval of aerosol height (e.g., Gabella et al., 1999; Koppers et al., 1997; Corradini and Cervino, 2006; Pelletier et al., 2008; Sanghavi et al., 2012; Frankenberg et al., 2012; Kokhanovsky and Rozanov, 2010) or cloud top height (e.g., Heidinger and Stephens, 2000; Preusker and Lindstrot, 2009; Fischer and Grassl, 1991; Rozanov and Kokhanovsky, 2004) have been published in the past. For a case study, Dubuisson et al. (2009) and Duforêt et al. (2007) exploited MERIS and POLDER data to derive aerosol height over oceans from reflectance ratios of channels inside and outside the $\mathrm{O}_{2} \mathrm{~A}$ band. Sanghavi et al. (2012) discussed using the $\mathrm{O}_{2} \mathrm{~A}$ and $\mathrm{O}_{2} \mathrm{~B}$ band to derive aerosol vertical distribution from SCIAMACHY data and applied the technique to a scene for 
Kanpur (India). However, for aerosols over land no operational data product exists to our knowledge, although hyperspectral measurements within the oxygen A band were and are performed by operational instruments such as SCIAMACHY, GOSAT, GOME, and GOME2. However, it is possible to derive the absorbing aerosol index (Torres et al., 1998; De Graaf et al., 2005) from such type of instruments, which is among other aerosol parameters also sensitive to aerosol height but does not retrieve quantitative aerosol vertical distribution parameters.

In the near and not too distant future, hyperspectral measurements within the oxygen A band will become widely available from instruments such as OCO2 (Haring et al., 2004; Crisp and Johnson, 2005), TROPOMI (Veefkind et al., 2012), Sentinel-4 (ESA, 2012), Sentinel-5 (ESA, 2012), or, if selected, ESA Earth Explorer (Bézy et al., 2008; Meijer et al., 2012) missions like FLEX (Clissold, 2008; Rascher et al., 2008; Stoll, 2003) or CarbonSat (Velazco et al., 2011). Operational aerosol height products are at least planned for TROPOMI on board the Sentinel 5 precursor (Veefkind et al., 2012; Sanders and de Haan, 2013) and for the Sentinels 4 and 5 (ESA, 2012). The aim of these products is to distinguish between aerosols in the planetary boundary layer and the free troposphere with desirably estimation of aerosol type, for example, to constrain surface concentrations of particulate matter (ESA, 2012). The specifications of these instruments vary widely with respect to spectral resolution, spatial resolution, temporal resolution, and signal to noise ratio (SNR), and each of these mission design parameters might have an impact on a possible retrieval of aerosol height.

Sanders and de Haan (2013) discussed the possible retrieval accuracy of aerosol height by propagating measurement and a priori errors through a locally linearized radiative transfer model using the framework of optimal estimation by Rodgers (2000). From the perspective of an operational retrieval, a positive result for the retrieval error with respect to given user requirements fulfills only a necessary condition. An actual retrieval must in addition converge robustly, treat multiple minima in the used cost function, deal with deviation of the real and the model atmosphere, and needs to be computationally fast enough to process and possibly reprocess large amounts of hyperspectral radiance data.

Aim of this paper is to propose the design and present an implementation of a fast and efficient forward operator for the simulation of hyperspectral top of atmosphere radiances in the oxygen A band, which is based on accurate radiative transfer simulations. The forward operator is based upon linear interpolation within a lookup table that was produced with efficient data compression using principal component analysis. Linear interpolation and computation of partial derivatives within this lookup table can then be performed in the much smaller space of expansion coefficients, which makes this approach computationally fast and, at the same time, memory efficient.
The generality of such a fast forward operator allows one to employ sophisticated optimal estimation techniques, as well as simple curve fitting techniques for the retrieval of forward operator parameters from measurements. Since the approach is computationally very efficient, it can be applied to operational retrievals, where in general huge amounts of data have to be processed with strict constraints on computational time. Here, the fast forward operator is applied in a simple inversion scheme using a curve fitting technique, which could represent a generic retrieval algorithm for the retrieval of aerosol height.

The fast forward operator is described in Sect. 2. Section 3 covers the setup of the sensitivity study, and the generation of synthetic measurements is introduced in Sect. 4. The inversion scheme is described in Sect. 5, and the the results of the sensitivity study are discussed in Sect. 6.

\section{Fast forward operator}

The following discussion is based on the assumption that the inversion of radiative transfer simulations for a complex atmosphere is the most suitable approach to retrieve aerosol height. Radiative transfer simulations numerically map an atmospheric state, which includes among others the quantities of interest, with simulated measurements. An inversion operator, which could include additional prior knowledge, can then be used as a retrieval operator. This assumption is emphasized, since this approach requires in general complex modeling, is prone to errors, requires precise knowledge about the optical properties of the atmosphere, the surface, and the aerosols, and can be computationally very demanding. To the best of our knowledge, no simpler approach for the retrieval of aerosol height exists up to now, as it was found, for example, for fluorescence emitted by vegetation, which can be retrieved using Fraunhofer lines without relying on radiative transfer (e.g., Frankenberg et al., 2011; Joiner et al., 2011).

The design of the fast forward operator was mainly driven by considerations about computational speed on standard computer hardware and simulation accuracy. This leads either to the use of the radiative transfer model directly as a forward model (e.g., compare the $\mathrm{OCO} 2$ retrieval by Bösch et al., 2006 or the retrieval of aerosol height from SCIAMACHY by Sanghavi et al., 2012), or to the use of the classical approach of a forward operator based on interpolation within a lookup table populated by radiative transfer calculations. The following discussion could also be based on neural networks, but the interpolation approach was chosen since its behavior is easier to understand and does not depend on tuned neural network parameters. 
Using a full-scale radiative transfer model as a forward operator entails some inherent advantages, as one does not rely on (linear) interpolation, which can introduce errors due to the nonlinear behavior of solutions of the radiative transfer equation. However, this approach will always be much more computationally demanding than a simple lookup table interpolation and thus might be unsuitable for application on standard computer hardware systems. A possible solution is to speed up the radiative transfer, which in general sacrifices simulation accuracy, which could repeal some of the advantages of this approach. On the other hand, populating a high-dimensional lookup table with hyperspectral radiative transfer simulations is also computationally expensive - especially if more and more state parameters are to be included. However, for the case of the oxygen A band, this obstacle can be overcome by exploiting the high correlation of the spectrum with respect to variations of the atmospheric state. Natraj et al. (2005) used principal component analysis to speed up the calculation of optical parameters for a radiative transfer model, while Hollstein and Lindstrot (2014) presented an approach based on principal component analyses, which can speed up the population of the lookup table by one or more orders of magnitude. Following the latter approach, a higher speedup is achieved for larger lookup tables. Hence, the computational aspect of filling up a potentially large lookup table can be considered as very well under control.

To summarize this approach - it was shown explicitly for the oxygen A band that a comparably small, randomly selected subset of spectra is sufficient to compute a set of principal components, which can be used to reconstruct the total lookup table, where the reconstruction accuracy is a function of the selected subset of spectra and the number of used principal components. Then, Hollstein and Lindstrot (2014) presented an algorithm that makes it possible to compute the expansion coefficients of a certain spectrum by simulating only a small subset of spectral channels. This implies that, for the oxygen A band, a relatively small number of spectral channels is sufficient to reconstruct the hyperspectral simulation, thus leading to an enormous speedup. This approach makes it feasible to increase the allowed computation time of the radiative transfer, for example, to increase simulation accuracy by using higher vertical and spatial resolution or by taking 3-D effects into account.

In addition to populating the lookup table, its possible huge size can become a major obstacle, especially for hyperspectral applications. As a consequence, the proposed lookup table for the fast forward operator exploits a data reduction technique based on principal component analysis. It is used to achieve effective data compression to reduce the size of the lookup table and also to gain computational speedups for the interpolation within that table.

The approach consists of two tables: one that stores the expansion coefficients of the simulated spectra with respect to the corresponding atmospheric state and a second one for the used principal components. Formally, let $\boldsymbol{x} \in \mathbb{R}^{n_{x}}$ be a state vector with $n_{x}$ free parameters for the radiative transfer model $R T(\boldsymbol{x})=\boldsymbol{y} \in \mathbb{R}^{n_{\lambda}}$, where $\boldsymbol{y}$ is the simulated spectrum and $n_{\lambda}$ is the number of simulated spectral channels. A generic lookup table would then consist of a possibly large set with $n$ states: $X=\left(\boldsymbol{x}_{1}, \ldots, \boldsymbol{x}_{n}\right)$ and a related set of simulated spectra $Y=\left(\boldsymbol{y}_{1}, \ldots, \boldsymbol{y}_{n}\right)$. Principal component analysis can be used to derive a number of $n_{p} \ll n_{\lambda}$ principal components $\boldsymbol{p}_{i} \in \mathbb{R}^{n_{\lambda}}, i=1, \ldots, n_{p}$, which can be combined in the principal component matrix $\mathbf{P}_{n_{p}} \in \mathbb{R}^{n_{p} \times n_{\lambda}}$ with $\left[\mathbf{P}_{n_{p}}\right]_{i j}=\left[\boldsymbol{p}_{i}\right]_{j}$. The expansion coefficients for each spectrum $\boldsymbol{c}_{i}=\mathbf{P} \times \boldsymbol{y}_{i}, i=1, \ldots, n$ can be computed such that a reconstructed spectrum $\tilde{y}_{i}$ can be expressed as $\tilde{y}_{i}=\mathbf{P}^{-1} \times$ $\boldsymbol{c}_{i}=\mathbf{P}^{T} \times \boldsymbol{c}_{i}$. The matrix $\mathbf{P}_{n_{p}}$ is orthonormal since the $p_{i}$ is pairwise orthonormal, such that $\mathbf{P}^{-1}=\mathbf{P}^{T}$. The reconstruction residual $\epsilon_{i}=\left|\boldsymbol{y}_{i}-\tilde{\boldsymbol{y}}_{i}\right|, i=1, \ldots, n$ for a single spectrum $y_{i}$ generally depends on the number of principal components and the reconstructed spectrum, while the mean reconstruction residual with respect to the total lookup table $\epsilon=$ mean $\left(\epsilon_{1}, \ldots, \epsilon_{n}\right)$ only depends on the number of principal components.

In general and with proper computation of $\mathbf{P}_{n_{p}}$, both residuals are strongly decreasing with increasing number of principal components; thus $n_{p}$ can be chosen such that the mean reconstruction error is sufficiently smaller than the measurement error of a possible instrument. As a result, the large table $Y$, which contains simulated spectra, can be replaced by the principal component matrix $\mathbf{P}_{n_{p}}$ and a table $C=\left(c_{1}, \ldots, c_{n_{l}}\right)$ with $c_{i}=\mathbf{P}_{n_{p}} \times \boldsymbol{y}_{i}$, which contains only the expansion coefficients for each spectrum and is smaller than $Y$ by a compression factor of $n_{\lambda} / n_{p}$.

To save large amounts of computation time for building the lookup table, the $c_{i}$ could be computed using the approach presented by Hollstein and Lindstrot (2014). The lookup table $C$ should be small enough to fit easily in the main memory of modern PCs. Not only does this approach save main memory when compared with an uncompressed table, it also saves much computation time since the interpolation and computation of partial derivatives can be computed in the space spanned by the expansion coefficients. This is possible since the compression from the principal component analysis is linear; thus compression and interpolation can be interchanged.

An interpolation scheme $I T(X, C, \boldsymbol{x})=\boldsymbol{c}$ in the expansion coefficient space must be implemented from which the desired interpolated spectrum can be computed by multiplying by the principal component matrix:

$\boldsymbol{y}(\boldsymbol{x})=\mathbf{P}_{n_{p}}^{T} \times I T(X, C, \boldsymbol{x})$.

This approach can be implemented to be computationally efficient and enables a simple path to exploit computation on modern GPGPUs (general purpose computation on graphics processing units). In an initialization step, the lookup table $C$ must be copied to the memory of the GPGPU, which is the slowest part, and then very little data transfer is needed since 
for a single interpolation only the two small vectors $\boldsymbol{x}$ and $\boldsymbol{c}$ need to be transferred from and to the main memory, where the final expansion to the desired spectra could be computed. The spectral resolution of the simulations can be adjusted easily and with little extra computational cost. When original simulations were performed with adequate spectral resolution, it is for many instruments sufficient to convolve the original principal components with the appropriate response functions to set up a fast forward operator for this instrument. This is numerically cheap, as the number of principal components is in general not too large and can be performed on the fly when the program is called. Similarly, spectral shifts as they occur, for example, for TANSO-FTS on board GOSAT can be corrected by convolving the principal components with response functions, which take the spectral shift into account. Further speedup in an inversion scheme can be achieved by selecting the number of used principal components with respect to the current value of the cost function. In the first steps of an iterative optimization scheme, it might be sufficient to reconstruct the spectra using only a few principal components, since it is sufficient to keep the reconstruction error well below the difference of actual measurement and simulation. The number of principal components can then be increased with decreasing cost function value to improve the reconstruction quality.

\section{Synthetic study setup}

Radiative transfer simulations were performed using the MOMO radiative transfer model (see Fell and Fischer, 2001; Hollstein and Fischer, 2012), which is a matrix operator model widely used at Freie Universität Berlin. Gaseous absorption was computed using line parameters from the HITRAN spectral database (Rothman et al., 2009) and a modified scheme to compute the K-distribution (Lacis and Oinas, 1991; Fu and Liou, 1992; West et al., 1990; Bennartz and Fischer, 2000). The parameter grid for the lookup table is shown in Table 1. The variation of the atmospheric state includes surface pressure, aerosol optical thickness, aerosol mean height, aerosol type, surface reflectance, and the viewing geometry. The full parameter range of the lookup table space is used throughout this study. The surface reflectance spectrum is assumed to be a linear function and is modeled using a reflectance value at $755 \mathrm{~nm}$ and at $780 \mathrm{~nm}$. The normalized aerosol vertical distribution $v(h, \mu, \sigma)$ with respect to height above the surface $h$ and mean height $\mu$ is modeled using a lognormal distribution with a width parameter of $\sigma=1.1$, which represents a narrow layer-like distribution:

$v(h, \mu, \sigma)=\frac{1 / \sqrt{2 \pi}}{h \cdot \log (\sigma)} \exp \left(-\frac{(\log (h)-\log (\mu))^{2}}{2 \log (\sigma)^{2}}\right.$.

The lognormal model can represent a wide range of possible aerosol vertical distributions, and a similar model was

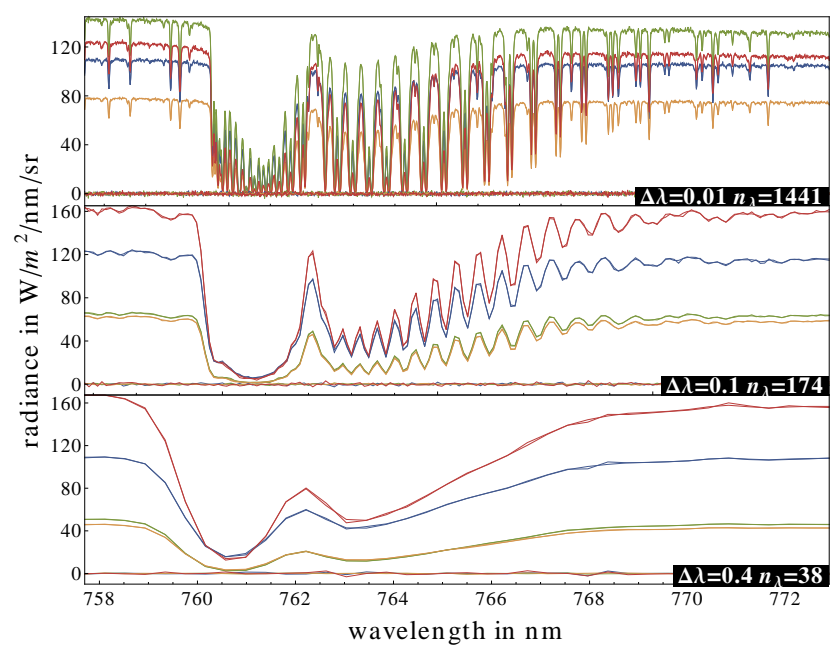

Figure 1. Random selection of synthetic spectra and inversion results for spectral resolution and sampling of $0.01 \mathrm{~nm}, 0.1 \mathrm{~nm}$, and $0.4 \mathrm{~nm}$. The synthetic signal to noise ratio was set to 100 (see Sect. 4 for a definition of the noise model), and the thin lines around the zero line show the fit residual.

also used by Sanghavi et al. (2012) in their previously cited case study.

As a result of this setup, the analysis is based on the retrieval of a single aerosol layer when only a single aerosol layer is present. A constant temperature profile is assumed throughout this discussion, since the actual profile should be given as background information. Introducing the temperature profile into this framework poses no specific difficulties and could be easily implemented as proposed by Lindstrot and Preusker (2012).

Several randomly selected spectra at three spectral resolutions are shown in Fig. 1. All spectra within the lookup table were simulated and could be used to compute the principal components, as well as evaluating the reconstruction performance for a specific set of principal components. The principal component algorithm provided by the Python package scikit-learn (Pedregosa et al., 2011) was used to compute the actual components. The first six components are shown in Fig. 2. Although it is in general unclear how to attribute specific physical properties to a single component, the first two components show a clear signature of the general oxygen absorption features and the linear model of the surface reflectance.

The uncompressed lookup table consists of 2496000 spectra with a spectral resolution of 4501 channels. Such a large amount of data makes the computation of the principal components for the complete data set numerically challenging. As shown by Hollstein and Lindstrot (2014), this is not necessary, since a randomly selected subsample of spectra of sufficient size is feasible to compute principal components that are valid for the complete lookup table. Since all spectra were simulated, it is possible to test the reconstruction 
Table 1. Parameter grid of the lookup table. The table contains $4 \times 5 \times 3 \times 5 \times 2 \times 2 \times 13 \times 8 \times 20=2496000$ states and corresponding spectra.

\begin{tabular}{rrl}
\hline Surface pressure $\rho$ & $n_{\rho}=4$ & $\rho=800 \mathrm{hPa}, 950 \mathrm{hPa}, 1013 \mathrm{hPa}, 1050 \mathrm{hPa}$ \\
Aerosol optical thickness & $n_{\tau}=5$ & $\tau=0.0,0.3,0.6,0.9,1.2$ \\
Aerosol center height & $n_{h}=3$ & $h=500 \mathrm{~m}, 2500 \mathrm{~m}, 4500 \mathrm{~m}$ \\
Aerosol type & $n_{t}=5$ & $t=1,2,3,4,5$ (dust, urban, continental, neutral, absorbing) \\
Surface reflectance at $755 \mathrm{~nm}$ & $n_{\alpha_{1}}=2$ & $\alpha_{1}=0.1,0.7$ \\
Surface reflectance at 780 $\mathrm{nm}$ & $n_{\alpha_{2}}=2$ & $\alpha_{2}=0.1,0.7$ \\
Viewing zenith angle $\mu$ & $n_{\mu}=13$ & $\mu=0.00,7.44,13.63,19.76,25.88,31.99,38.10,44.21,50.32$, \\
& & $56.42,62.53,68.63,74.74 \mathrm{in} \mathrm{deg}$ \\
Solar zenith angle $\mu_{\mathrm{S}}$ & $n_{\mu_{\mathrm{S}}=8}$ & $\mu_{\mathrm{S}}=0.00,7.44,13.63,19.76,25.88,31.99,38.10,44.21 \mathrm{in} \mathrm{deg}$ \\
Relative azimuth angle $\phi$ & $n_{\phi}=20$ & $\phi=0.00,9.47,18.95,28.42,37.89,47.37,56.84,66.32,75.79$, \\
& & $85.26,94.74,104.21,113.68,123.16,132.63,142.11,151.58$, \\
& & $161.05,170.53,180.00 \mathrm{in} \mathrm{deg}$ \\
\hline
\end{tabular}

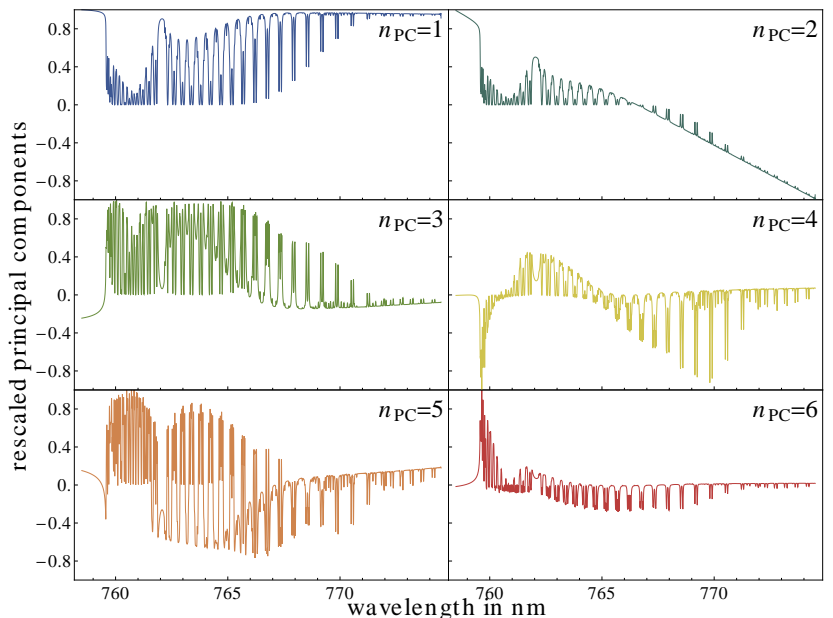

Figure 2. The first six principal components of the principal component matrix $P_{n_{p}}$. The spectra are normalized for better graphical representation.

accuracy of the total data set with respect to the number of used principal components.

Figure 3 shows the dependency of the mean of the synthetic signal to noise ratio for the whole lookup table with respect to the number of used principal components. The synthetic signal to noise level was defined as

$\operatorname{SNR}=\operatorname{mean}(\boldsymbol{y}) / \operatorname{SD}(\boldsymbol{y}-\tilde{\boldsymbol{y}})$,

where $\boldsymbol{y}$ is a synthetic measurement and $\tilde{\boldsymbol{y}}$ a reconstructed spectrum and is used as a measure of fit quality throughout this paper.

The results clearly show that the reconstruction quality increases strongly with increasing number of principal components and that a number of 10 principal components is sufficient to represent the original data with a mean synthetic SNR of approximately 1000 .

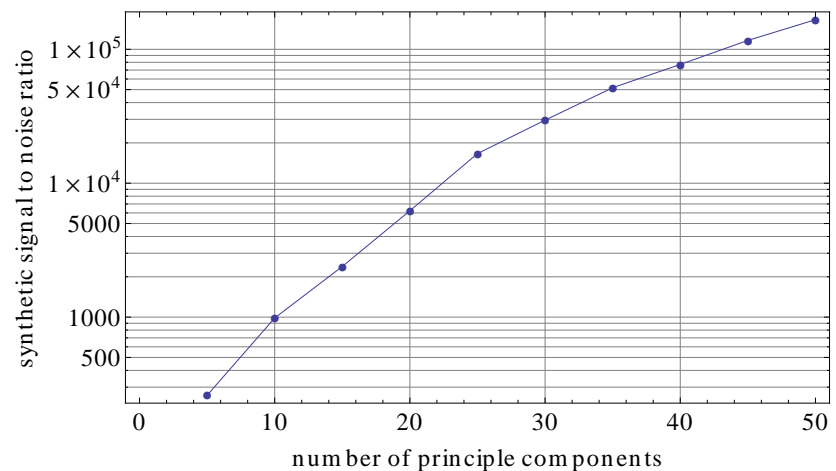

Figure 3. Mean synthetic SNR with respect to the number $n_{p}$ of principal components.

Histograms of the reconstruction error for increasing values of $n_{p}$ are shown in Fig. 4. The results show that the reconstruction quality increases dramatically with increasing number of principal components. The histogram for 15 principal components shows a peculiar dip in the middle of the distribution, which sets this distribution slightly apart from the other ones, but causes no difficulties since the whole distribution shows a much better mean reconstruction quality than when using only five principal components. This effect shows that it is in principle difficult to establish a physical link between a principal component and its effect on the overall reconstruction accuracy when it is added to the reconstruction matrix.

The aerosol optical models were implemented according to Levy et al. (2007). These models are used by the MODIS aerosol retrieval and were specifically designed to fit observations for different locations on the globe. These models are representative of a MODIS perspective, since they were designed to represent what can be retrieved with MODIS and not for what could be seen in the oxygen A band. It is beyond the scope of this paper to derive an aerosol model that maximizes the use of all the information in the oxygen A band, 


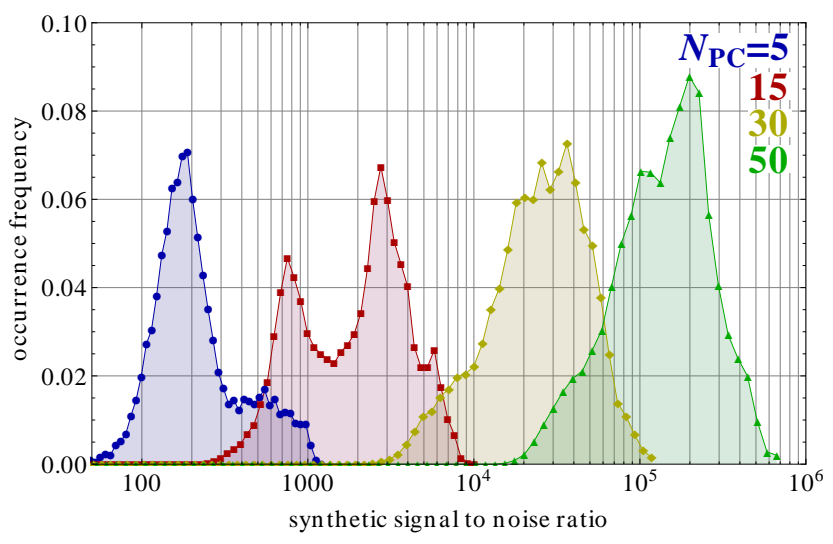

Figure 4. Histograms of the synthetic SNR for selected values of $n_{p}$. Note that the abscissa is in log scale and that occurrence bins were chosen to be equally spaced in a log plot.

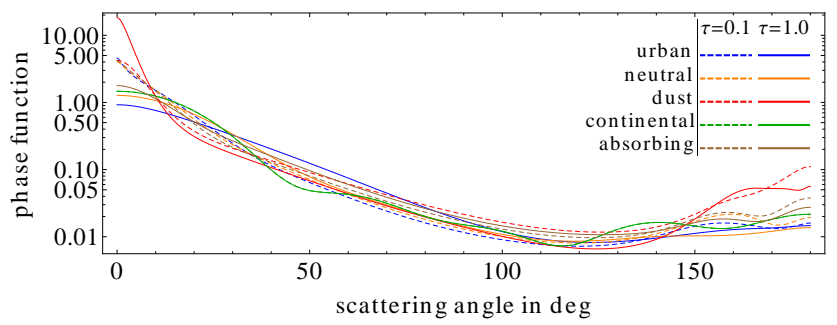

Figure 5. Phase functions at $774.5 \mathrm{~nm}$ of the five used aerosol models.

but these models are a good choice nevertheless. They allow the assessment of which of these widely used models can be distinguished from the discussed type of measurements. From the published optical properties, the urban, neutral, dust, continental, and absorbing types were implemented and Mie calculations using the implementation provided by Wiscombe (1980) were used to compute phase functions, extinction cross sections, and single scattering albedo. Aerosol phase functions at $774.5 \mathrm{~nm}$ with respect to scattering angle and optical thickness are shown in Fig. 5.

The approach of this study is to expand on the retrieval error analysis as presented by Sanders and de Haan (2013) and to implement an actual retrieval scheme, where simulated measurements with realistic random errors are fitted using the fast forward operator. This approach is suited to realistically discuss the difficulties that can arise from multiple minima in the cost function, which can be caused by almost linearly dependent partial derivatives of the forward operator.

The dependency of the partial derivative of the forward operator with respect to aerosol optical thickness and aerosol height is shown in Fig. 6 and for surface reflectance and aerosol optical thickness in Fig. 7. The partial derivatives were multiplied by a scaling factor with appropriate unit, such that both rescaled spectra carry the unit radiance and

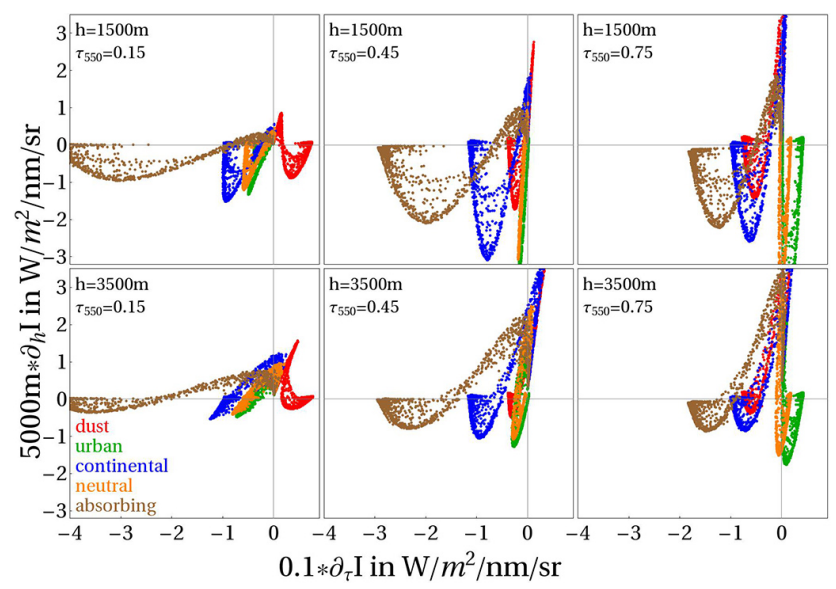

Figure 6. Several scatterplots of the partial derivative spectrum of the fast forward operator with respect to aerosol height and aerosol optical thickness. Both partial derivatives were scaled with a factor with appropriate unit, such that the unit of the resulting quantity is radiance and that both spectra have similar magnitude. Shown in the top left of each panel is the base aerosol optical thickness and aerosol height. Different colors indicate results for the different aerosol types. From the left to right panel, the aerosol height is kept constant and the aerosol optical thickness increases, while from top to bottom panel the aerosol height is changing. The baseline parameters are solar angle $\mu_{\mathrm{S}}=10^{\circ}$, viewing angle $\mu=20^{\circ}$, and relative azimuth angle $\varphi=30^{\circ}$; surface reflectance is $\alpha=0.2$, and surface pressure was set to $\rho=1013 \mathrm{hPa}$.

are of comparable magnitude. By neglecting non-linearity, one can interpret the scaling factors as the specific quantity for both compared parameters, which causes a similar radiance change.

Figure 6 shows that the partial derivatives with respect to aerosol height and aerosol optical thickness are quite variable functions of the aerosol model and can be largely described by almost linear sections with alternating signs of the slope. Figure 6 shows the partial derivatives with regard to aerosol height and aerosol optical thickness, which rather strongly depend on the aerosol model and can be largely described by almost linear sections with alternating signs of the slope. This behavior could potentially lead to multiple minima in the least squares cost function. Also, their differences could help to discriminate between aerosol types. Figure 7 shows that the partial derivatives with respect to aerosol optical thickness and surface reflectivity are strongly linearly correlated, with a strong dependency on aerosol type. This indicates one of the main theoretical problems with deriving the aerosol height from the oxygen A band alone. Crucial for the retrieval accuracy will be the proper discrimination of surface reflectivity and aerosol optical depth. Both parameters are highly variable in space and time on short scales, such that prior knowledge with sufficiently small uncertainty will likely be unavailable. 


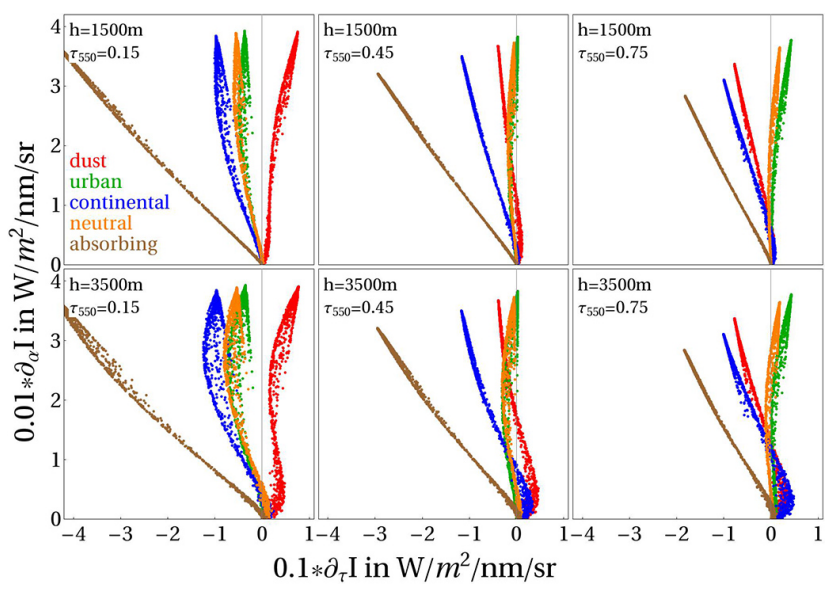

Figure 7. Similar setup to that shown in Fig. 7, but the partial derivatives with respect to surface reflectivity and aerosol optical thickness are compared.

\section{Synthetic measurements}

Within the scope of this paper is the simulation of general hyperspectral radiometers with arbitrary spectral resolution, spectral sampling, and signal to noise level. As generic noise model, a random noise vector $\boldsymbol{n}$ was added to a forward operator simulation to produce a synthetic measurement $\boldsymbol{y}_{m}$ for the oxygen A band:

$\boldsymbol{y}_{m}=\boldsymbol{y}+\boldsymbol{n}(\boldsymbol{y}), \boldsymbol{n}(\boldsymbol{y})=\boldsymbol{r}_{-1,1}$ mean $(\boldsymbol{y}) / \mathrm{SNR}$,

where $\boldsymbol{r}_{-1,1}$ is a vector with the dimension of the simulations, which contains random numbers between -1 and 1 , SNR is the prescribed signal to noise ratio, and mean $(y)$ denoted the spectral average for the complete oxygen A band. With this definition, the synthetic SNR of a spectral fit is approximately the prescribed SNR number.

A more realistic model can be used with a specific type of instrument in mind, but would, at the same time, be less general for other types of instruments. This makes the setup of a generic noise model for this type of study difficult. Although the chosen model is very simple and generic, it is sufficiently close to a realistic noise model (e.g., see Aiazzi et al., 2006) and avoids the problem of almost zero relative error for small measurements when using pure multiplicative noise. In addition, the model is controlled by a single parameter, which makes the discussion of the noise more coherent.

\section{Inversion scheme}

The fast forward operator was implemented in FORTRAN, parallelized using OpenMP, and run on a standard desktop computer with $8 \mathrm{~GB}$ of main memory and an Intel i7-3770 $\mathrm{CPU}$ with 4 cores running at $3.2 \mathrm{GHz}$. The multi-linear interpolation in all dimensions of the lookup table was implemented in FORTRAN in such a way that the first-order
Table 2. Benchmark results of the fast forward operator on an Intel i7-3770 with respect to the number of used principal components $n_{p}$, spectral resolution SR, spectral sampling SS, and for the pure interpolation result $\boldsymbol{y}$ and the interpolation result and corresponding Jacobian $\boldsymbol{y}, \nabla y$. Spectral resolution and spectral sampling were chosen to be the same per run and carry the unit of $\mathrm{nm}$. The unit for the benchmark results is simulated spectra per second. Eight threads were used for the multithread run.

\begin{tabular}{lllllll}
\hline & & \multicolumn{2}{c}{ Single thread } & & \multicolumn{2}{c}{ Multi-thread } \\
\cline { 3 - 4 } \cline { 7 - 8 } SR $=$ SS & $n_{p}$ & $\boldsymbol{y}$ & $\boldsymbol{y}, \nabla y$ & & $\boldsymbol{y}$ & $\boldsymbol{y}, \nabla y$ \\
\hline 0.1 & 5 & 5780 & 1580 & & 26694 & 7213 \\
0.01 & 5 & 5777 & 1574 & & 26734 & 7221 \\
0.001 & 5 & 5768 & 1581 & & 26694 & 7222 \\
\hline 0.1 & 15 & 4129 & 845 & & 20100 & 4072 \\
0.01 & 15 & 4125 & 845 & & 19997 & 4089 \\
0.001 & 15 & 4120 & 844 & & 20141 & 4105 \\
\hline 0.1 & 30 & 3020 & 500 & & 14213 & 2325 \\
0.01 & 30 & 2974 & 497 & & 13862 & 2343 \\
0.001 & 30 & 3002 & 501 & & 13866 & 2402 \\
\hline
\end{tabular}

derivatives, which we will call Jacobians throughout this paper, are computed analytically from the interpolation formula. This approach is numerically stable and needs only little extra computational time.

Results for a simple benchmark are shown in Table 2. Each benchmark result is based on the computation of 50000 randomly selected states. The benchmark shows that the run time of the forward operator is almost independent of spectral resolution and sublinearly increases with increasing number of principal components.

The Levenberg-Marquardt optimization routine lmder from the MINIPACK project provided by Moré et al. (1984) was used to minimize the sum of least squares between simulated measurements and results of the fast forward operator. As discussed in Sect. 3, no prior knowledge is assumed since, for the crucial variables aerosol type, aerosol height, aerosol optical thickness, and surface reflectance, prior knowledge will likely not be available with sufficiently small error.

The original lmder routine is implemented for an unbounded problem and was modified to ensure that the minimization search stays within the bounds of the lookup table. This is achieved by modifying a computed next iteration step such that the bounds of the lookup table can only be reached asymptotically. If an actual computed step were to cross the lookup table boundary, the computed step size would be replaced by half of the distance between the actual position and the boundary. Finally, a certain minimum step size for aerosol optical depth was chosen as convergence criterion for the iteration. 
The lmder routine is called 20 times with randomly selected starting values for each inversion attempt, and the best result in terms of cost function value is returned as a final result. This approach eliminates effects caused by possible multiple minima in the cost function, or a sensitivity of the inversion for the starting values.

\section{Synthetic retrieval results}

The sensitivity study is based on a Monte Carlo approach. A total of 50000 retrievals with randomly selected state vectors are performed for a given setting of spectral resolution, spectral sampling, signal to noise ratio, prior aerosol type information, and surface inhomogeneity. The complete parameter space of the lookup table as given in Table 1 was used for the generation of random atmospheric states. Surface inhomogeneity is modeled by applying the independent column approach, where the mean value over $N_{\alpha}=9$ simulations using a range of different surface reflectances is taken. The resulting set of prescribed state vectors and inversion results is then analyzed by means of scatterplots and mean values.

Two named cases are distinguished throughout the analysis: the best case and the realistic case scenario. The best case scenario is characterized by a retrieval with known aerosol type and simulations with homogeneous surface reflectance, while for the realistic case the aerosol type is a free inversion parameter and surface inhomogeneity is taken into account. The two cases can be used to study the effect of an unknown aerosol type, which is believed to have only minor effects (cf. Sanders and de Haan, 2013), and also the effect of the finite spatial resolution of a hypothetical sensor. In general, spectral resolution, spectral sampling, and spatial resolution are competing factors for an instrument with given external constraints. Increasing the spectral resolution decreases the amount of energy within each channel, which can be compensated with more sensitive detectors, longer integration time, or a larger footprint on the surface of the earth. The realistic case is therefore more realistic for hyperspectral instruments such as TROPOMI or TANSO-FTS, where an instrument with lower spectral resolution such as FLEX offers much higher spatial resolution.

Spectral resolution and sampling are two free and independent parameters of the fast forward operator setup, and their effects can be studied independently. Here, we focus our analysis on the retrieval of aerosol parameters and simplify the parameter space to cases where spectral resolution and sampling are equal. This implies that no oversampling is considered, which can help to reduce the impact of random noise for real instruments.

It is of great importance for the general applicability of the inversion results that the scheme robustly finds the global minimum of the cost function. This is ensured by a large number of random starting values and a comparison of the resulting synthetic SNR value from the fit residual with the

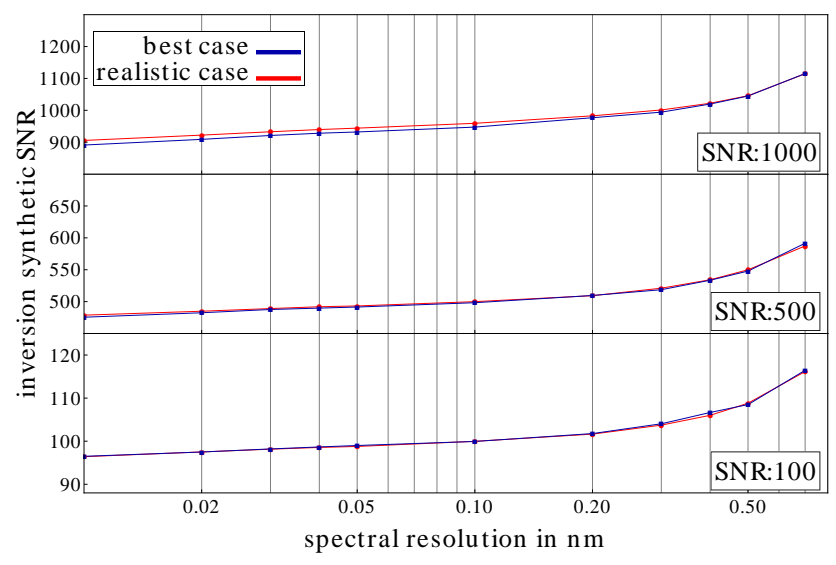

Figure 8. Mean synthetic signal to noise level from inversion attempts vs. spectral resolution for three prescribed SNR levels. Blue lines indicate the best case setup, whereas results for the realistic case are shown using red color. Spectral sampling is equal to the spectral resolution. The best case is characterized by a homogeneous surface and known aerosol model, where the realistic case is characterized by an unknown aerosol model and a heterogeneous surface reflectivity.

prescribed one. If the achieved residual is on the order of the prescribed noise, the inversion is successful and different schemes might only be more efficient in the needed computational burden or memory use. Figure 8 shows the mean inversion signal to noise ratio from the fit residual with respect to spectral resolution, prescribed signal to noise ratio, and the best case and realistic case scenario. The results show clearly that the inversion succeeds and is capable of finding a minimum in the cost function, which can be completely explained by noise. This is also highlighted in Fig. 1, where several simulated spectra, inversion results, and resulting residuals are shown for three spectral resolutions. The general inversion residual is within the prescribed noise, which indicates that an improvement above the results presented here is only possible by introducing further measurements or additional prior knowledge. However, such an analysis is beyond the scope of this paper.

As discussed above, the methodology of this study is Monte Carlo-like, and an overview about results for the best case, realistic case, synthetic SNR value of 500, and varying spectral resolution is shown in Fig. 9. The top row of subfigures shows the effect of unknown aerosol type and surface inhomogeneity for constant signal to noise ratio, spectral resolution, and spectral sampling. While the results for the aerosol height retrieval even slightly improve, the retrieval of aerosol optical depth is affected by relaxing these constraints. The aerosol height retrieval is almost unaffected, its bias is slightly reduced and the slope is closer to one. The scatter of aerosol optical depth retrieval increases, and the slope deviates more from one. In this respect, the aerosol height retrieval is more stable with respect to aerosol type 


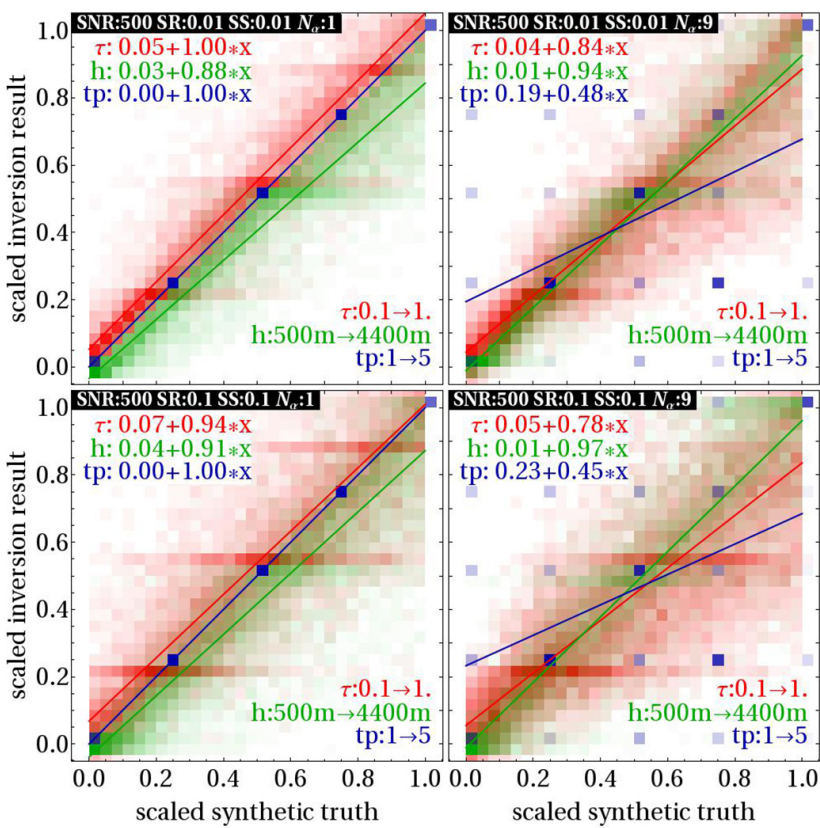

Figure 9. Overview about inversion runs for various scenarios. Each figure is a combined scatterplot of a retrieved and prescribed parameter for 10000 randomly selected cases. A scenario is defined by signal to noise level, spectral width, spectral sampling, number of surface reflectances, and aerosol type background information. These background settings are shown in the top left of each figure, and an SNR value of 500 was used here. Also in the top left shown are line parameters for a linear fit per parameter, and the true parameter interval is shown in the bottom right. Shown are retrieval results for the aerosol optical thickness (red), aerosol height (green), and aerosol type (blue). No interpolation between aerosol types was used, and types were ordered like in Table $1(0.0=$ dust, $0.25=$ urban, $0.5=$ continental, $0.75=$ neutral, $1.0=$ absorbing $)$. The left column shows results for the the best case (aerosol type information known, homogeneous surface with $N_{\alpha}=1$ ) and the right column for the realistic case (unknown aerosol type, inhomogeneous surface with $N_{\alpha}=9$ ) scenario. From top to bottom the spectral resolution (SR) and sampling (SS) are decreased from $0.01 \mathrm{~nm}$ (top) to $0.1 \mathrm{~nm}$ (bottom). The color scale follows an inverse power distribution to enhance the visibility of low-density bins.

and surface inhomogeneity than the retrieval of aerosol optical depth. The bottom row of the figure shows the effect of decreasing the spectral resolution and spectral sampling to $0.1 \mathrm{~nm}$. As for the case with higher spectral resolution and sampling, the retrieval of aerosol height is more stable, while the retrieval of aerosol optical depth is more strongly affected.

The presented scatterplots can be seen as a best estimate for the validation of the retrieval scheme in reality. Its success can be measured in terms of absolute accuracy and relative to given user requirements, which in general strongly depend on a specific application. Aim of this paper is to be rather general and not to base the discussion on a certain user

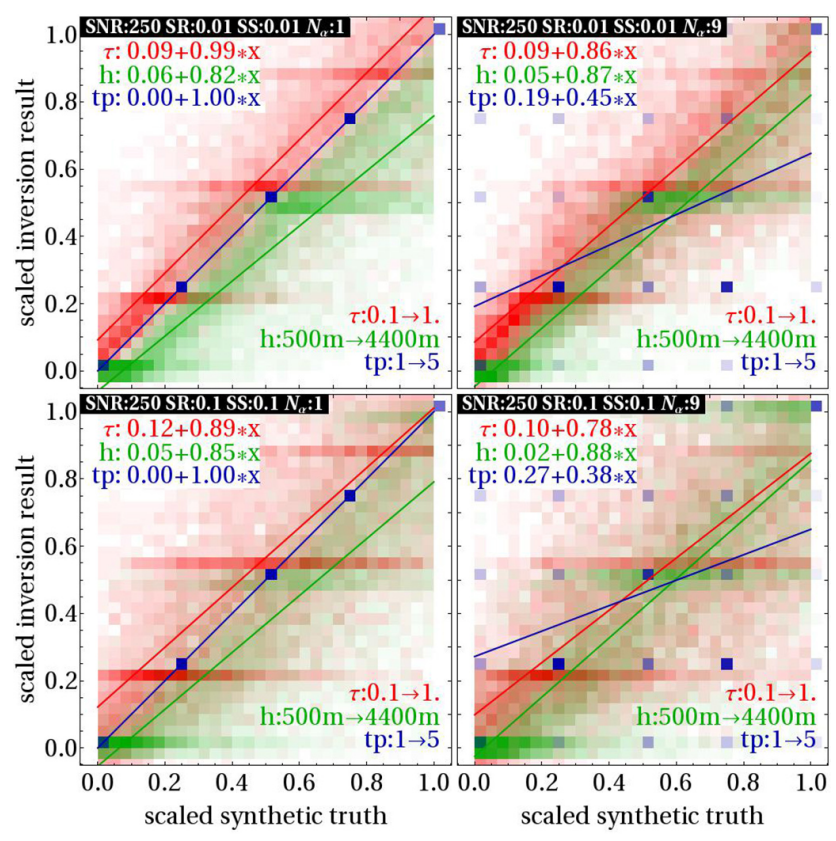

Figure 10. Similar to Fig. 9 but for a prescribed signal to noise ratio of 250 .

requirement and application. Hence, throughout this paper the absolute retrieval accuracy is taken as a measure.

Visible in all scatterplots are minor artifacts that are caused by the grid points of the tabulated aerosol optical depth and aerosol height. The artifacts are horizontal lines of increased occurrence of a parameter value, which is a grid point in the lookup table (compare also with Table 1). An actual retrieval could simply avoid these artifacts by using a finer grid in the lookup table, which would then better represent the nonlinear response of the simulations with respect to these parameters. These artifacts are shown in Figs. 9 and 10 to highlight the effects of a finite resolution in the lookup table, but they are excluded in the further analysis.

Similar scatterplots, but for a prescribed signal to noise ratio of 250, are shown in Fig. 10. The general distribution of scatter points is much wider than in Fig. 9, although the regression lines are only slightly affected. It will depend on the desired application whether such scatter can be accepted.

In contrast to other shown inversion results, each subfigure of Figs. 9 and 10 is based on the inversion of 10000 randomly selected state vectors. Its computation on a standard PC with Intel i7-3770 CPU took approximately $30 \mathrm{~min}$ (see also the benchmark results in Sect. 5). Each inversion for a single spectrum was repeated 20 times with randomly selected starting values to ensure convergence to the global minimum of the least squares cost function.

The discussed results provide evidence that the retrieval of aerosol height will depend on instrumental parameters like spectral resolution, spectral sampling, and signal to noise ratio, as well as on the scene itself. This is included in the 


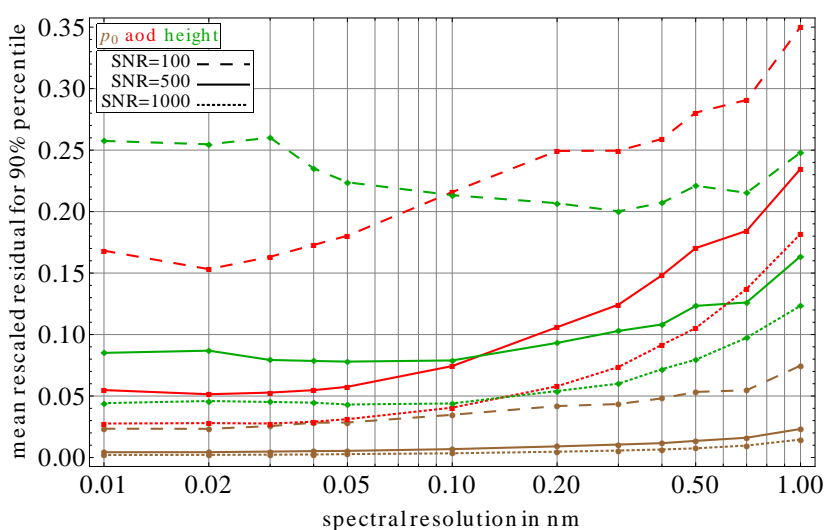

Figure 11. Inversion success of surface pressure (cyan), aerosol optical depth (red), and aerosol height (green) with respect to spectral resolution and prescribed signal to noise ratio (dashed, solid, dot dashed). Shown is the mean absolute residual of the $90 \%$ best inversion results in rescaled units (compare with Figs. 9 and 10). Spectral sampling for each point is equal to spectral resolution. The realistic case background settings were used.

discussion by prescribing or removing the aerosol type information and introducing surface inhomogeneities. Results regarding this assumption are shown in Fig. 11, which compiles the inversion success for surface pressure, aerosol optical thickness, and aerosol height. Inversion success was defined as the mean absolute radiance residual for the $90 \%$ best cases in rescaled units as they were used in Figs. 9 and 10. Zero indicates a perfect mean inversion, while one indicates that the mean residual is on the order of the maximum of the range of the retrieved quantity. Surface pressure is shown merely as a reference; its retrieval success depends almost only on signal to noise error and decreases only slightly with increasing spectral resolution.

The results for aerosol optical depth and aerosol height behave quite differently. While being sensitive to spectral resolution, the inversion success strongly depends on the signal to noise level. The strongest increase for aerosol optical thickness can be seen when decreasing the spectral resolution from $1 \mathrm{~nm}$ to $0.1 \mathrm{~nm}$. From that on, only minor improvements in the retrieval can be achieved by increasing the spectral resolution of the instrument. Again, it will depend on user requirements whether a possibly small gain in retrieval accuracy from increasing the spectral resolution is feasible. A similar but weaker behavior can be seen for the retrieval of aerosol height. Depending on the signal to noise level, the retrieval of aerosol height could become worse for increased spectral resolution. It should be noted that these results describe the inversion success with respect to spectral resolution at constant signal to noise range. When increasing the spectral resolution, one automatically increases the dynamic range of the signal since the fine oxygen absorption lines become better and better resolved. While these features carry information about the aerosol height, they are strongly

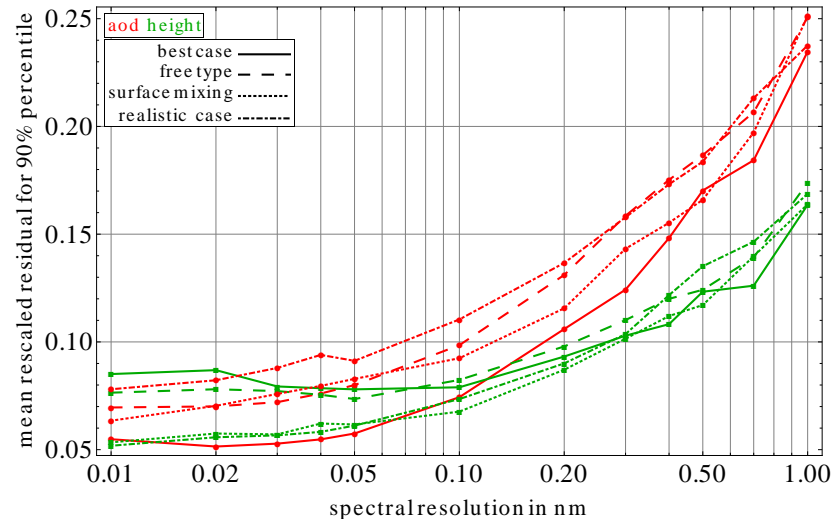

Figure 12. Similar to Fig. 11 but only for aerosol optical thickness and aerosol height, but with respect to aerosol type information and surface inhomogeneity. Shown are the best case and the realistic case scenario and also the free type scenario where the aerosol type is unknown to the retrieval but the surface is homogeneous and also the surface mixing scenario where the aerosol type information is given but the surface is assumed to be inhomogeneous.

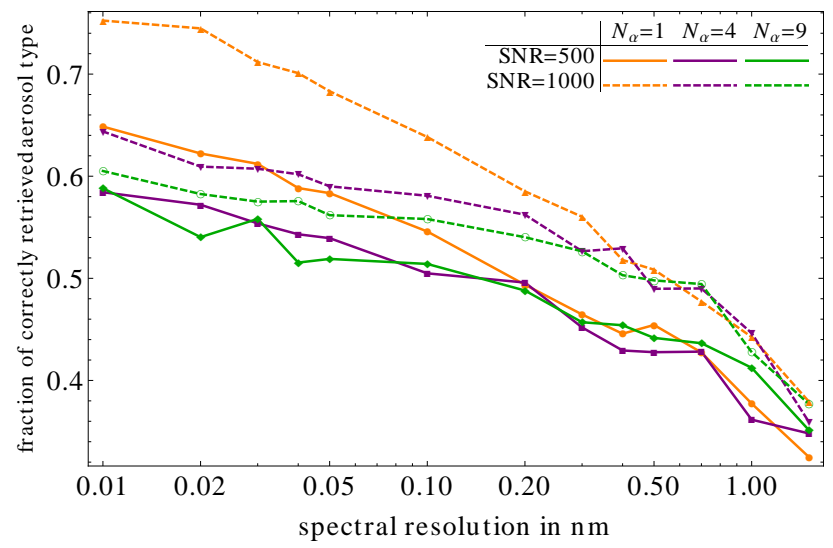

Figure 13. Fraction of correctly retrieved aerosol type with respect to spectral resolution, prescribed signal to noise ratio (solid/dashed lines), and surface inhomogeneity (orange, purple, and green color).

affected by noise, which can lead to a decrease in retrieval accuracy. For a real instrument, increasing the spectral resolution will affect the signal to noise level or other parameters such as spatial resolution.

For the shown results, the realistic case background information parameters were used. Figure 12 shows the behavior of the aerosol type and aerosol height with respect to spectral resolution, aerosol type information, and surface inhomogeneity. All combinations of known/unknown aerosol type and homogeneous/inhomogeneous surface are shown. The overall effect is minor and is more pronounced for the aerosol optical thickness. A conclusion is that the retrieval of aerosol height is robust against variations in aerosol type and surface homogeneity. This conclusion is valid for the total physical space that has been discussed here. It could be 
exploited in much more detail, for example, by analyzing it with respect to specific aerosol optical parameters and surface conditions, but this is left as subject for future research.

Although beyond the scope of this paper and likely of any aerosol retrieval based purely on the oxygen A band, the presented scheme allows the investigation of to what extent possible aerosol type information can be retrieved. Results of such an analysis are shown in Fig. 13, which shows the fraction of correctly retrieved aerosol type with respect to spectral resolution, prescribed signal to noise ratio, and surface inhomogeneity. In general, the fraction of correctly retrieved aerosol type is increasing with increasing spectral resolution and is decreasing with increasing signal to noise ratio and increasing surface inhomogeneity. Although far from being the best approach of retrieving the aerosol type, hyperspectral radiance measurements in the oxygen A band could potentially contribute to a better retrieval of aerosol type.

\section{Conclusions}

A fast forward operator for the simulation of hyperspectral radiances in the oxygen A band was described, benchmarked on a standard computer, and applied for a sensitivity study concerning the retrieval of aerosol optical depth and aerosol height. Study parameters were spectral resolution, spectral sampling, signal to noise ratio, aerosol type information, and surface inhomogeneity. The study showed that the retrieval generally benefits from higher spectral resolution, with the strongest increase in retrieval accuracy above a spectral resolution of $0.1 \mathrm{~nm}$. Signal to noise ratio strongly affects the retrieval and is a key parameter when designing an instrument and a retrieval scheme. The retrieval of aerosol height seems to be robust even when aerosol type information is missing and when surface inhomogeneity is introduced. These conclusions hold for the retrieval of a single aerosol layer when a single aerosol layer is present. More complex vertical profiles should be subject to future research. Evidence was found that the oxygen A band contains valuable information about the aerosol type, which could be used in an aerosol retrieval that utilizes additional spectral bands. The dependency with respect to the temperature profile was not discussed here and might be subject to future research.

Acknowledgements. We wish to thank our colleague Rasmus Lindstrot for fruitful discussions and for his help when preparing the manuscript. We thank the two anonymous reviewers, whose comments helped to improve and clarify this manuscript.

Edited by: A. Kokhanovsky

\section{References}

Aiazzi, B., Alparone, L., Barducci, A., Baronti, S., Marcoionni, P., Pippi, I., and Selva, M.: Noise modelling and estimation of hyperspectral data from airborne imaging spectrometers, Ann. Geophys., 49, doi:10.4401/ag-3141, 2006.

Amiridis, V., Tsekeri, A., Marinou, E., Wandinger, U., Kazadzis, S., Giannakaki, E., Mamouri, R., Kokkalis, P., and Herekakis, T. Lidar Climatology of Vertical Aerosol Structure for Space-Based Lidar Simulation Studies, Final Report, herekakisESA-ESTEC Contract RFQ/3-13301/11/NL/FF/fk, 2013, available at: http:// lidar.space.noa.gr:8080/livas/, 2013.

Amodeo, A., Pappalardo, G., Bösenberg, J., Ansmann, A., Apituley, A., Alados-Arboledas, L., Balis, D., Böckmann, C., Chaikovsky, A., Comeron, A., Freudenthaler, V., Gustaffson, O., Hansen, G., Mitev, V., Nicolae, D., Papayannis, A., Perrone, M., Pietruczuk, A., Pujadas, M., Putaud, J., Ravetta, F., Rizi, V., Simeonov, V., Spinelli, N., Stoyanov, D., Trickl, T., and Wiegner, M.: A European research infrastructure for the aesorol study on a continental scale: EARLINET-ASOS., Proceedings of the SPIE 6367, 674567450Y, 2007.

Andreae, M. O.: Soot Carbon and Excess Fine Potassium: LongRange Transport of Combustion-Derived Aerosols, Science, 220, 1148-1151, doi:10.1126/science.220.4602.1148, 1983.

Bennartz, R. and Fischer, J.: A modified k-distribution approach applied to narrow band water vapour and oxygen absorption estimates in the near infrared, J. Quant. Spectr. Radiat. Trans., 66, 539-553, 2000.

Betzer, P. R., Carder, K. L., Duce, R. A., Merrill, J. T., Tindale, N. W., Uematsu, M., Costello, D. K., Young, R. W., Feely, R. A., Breland, J. A., Bernstein, R. E., and Greco, A. M.: Long-range transport of giant mineral aerosol particles, Nature, 336, 568571, doi:10.1038/336568a0, 1988.

Bézy, J.-L., Bensi, P., Berger, M., Carnicero, B., Davidson, M., Drinkwater, M., Durand, Y., Héliere, F., Ingmann, P., Langen, J., Lin, C., Meynart, R., Rebhan, H., Silvestrin, P., and A.Thompson: ESA Future Earth Observation Explorer Missions, Proc. of SPIE, 7081, 70810S, 2008.

Bösch, H., Toon, G. C., Sen, B., Washenfelder, R. A., Wennberg, P. O., Buchwitz, M., de Beek, R., Burrows, J. P., Crisp, D., Christi, M., Connor, B. J., Natraj, V., and Yung, Y. L.: Space-based near-infrared $\mathrm{CO}_{2}$ measurements: Testing the Orbiting Carbon Observatory retrieval algorithm and validation concept using SCIAMACHY observations over Park Falls, Wisconsin, J. Geophys. Res. Atmos., 111, D23302, doi:10.1029/2006JD007080, 2006.

Chin, M., Kahn, R. A., and Schwartz, S. E. (Eds.): CCSP 2009: Atmospheric Aerosol Properties and Climate Impacts, A Report by the US Climate Change Science Program and the Subcommittee on Global Change Research, National Aeronautics and Space Administration, Washington, DC, USA, 2009.

Clarke, A. D. and Noone, K. J.: Soot in the Arctic snowpack: a cause for perturbations in radiative transfer, Atmos. Environ. (1967), 19, 2045-2053, doi:10.1016/0004-6981(85)90113-1, 1985.

Clissold, P. (Ed.): ESA SP-1313/4 Candidate Earth Explorer Core Missions - Reports for Assessment: FLEX - FLuorescence EXplorer, ESA Communication Production Office, 2008.

Corradini, S. and Cervino, M.: Aerosol extinction coefficient profile retrieval in the oxygen A-band considering multiple scattering atmosphere. Test case: SCIAMACHY nadir simulated 
measurements, J. Quant. Spectr. Radiat. Transf., 97, 354-380, doi:10.1016/j.jqst.2005.05.061, 2006.

Crisp, D. and Johnson, C.: The orbiting carbon observatory mission, Acta Astronautica, 56, 193-197, doi:10.1016/j.actaastro.2004.09.032, iAA International Symposium on Small Satellites for Earth Observation, 2005.

De Graaf, M., Stammes, P., Torres, O., and Koelemeijer, R.: Absorbing Aerosol Index: Sensitivity analysis, application to GOME and comparison with TOMS, J. Geophys. Res. Atmos., 110, D01201, doi:10.1029/2004JD005178, 2005.

Dubuisson, P., Frouin, R., Dessailly, D., Duforêt, L., Léon, J.-F., Voss, K., and Antoine, D.: Estimating the altitude of aerosol plumes over the ocean from reflectance ratio measurements in the O2 A-band, Remote Sens. Environ., 113, 1899-1911, doi:10.1016/j.rse.2009.04.018, 2009.

Duforêt, L., Frouin, R., and Dubuisson, P.: Importance and estimation of aerosol vertical structure in satellite ocean-color remote sensing, Appl. Opt., 46, 1107-1119, 2007.

ESA: GMES sentinels 4 and 5 mission requirements traceability document, issue 1, revision 0, Tech. rep., European Space Research and Technology Centre, available at: http://esamultimedia.esa.int/docs/EarthObservation/S4_5_5p_ MRTD_issue_1.0_authorised.pdf (last access: 22 October 2013), 2012.

Fell, F. and Fischer, J.: Numerical simulation of the light field in the atmosphere-ocean system using the matrix-operator method, J. Quant. Spectr. Radiat. Trans., 69, 351-388, doi:10.1016/S00224073(00)00089-3, 2001

Fischer, J. and Grassl, H.: Detection of cloud-top height from backscattered radiances within the oxygen A band. Part 1: Theoretical study, J. Appl. Meteorol., 30, 1245-1259, 1991.

Frankenberg, C., Butz, A., and Toon, G. C.: Disentangling chlorophyll fluorescence from atmospheric scattering effects in $\mathrm{O} 2$ A-band spectra of reflected sun-light, Geophys. Res. Lett., 38, L03801, doi:10.1029/2010GL045896, 2011.

Frankenberg, C., Hasekamp, O., O’Dell, C., Sanghavi, S., Butz, A., and Worden, J.: Aerosol information content analysis of multiangle high spectral resolution measurements and its benefit for high accuracy greenhouse gas retrievals, Atmos. Meas. Tech., 5, 1809-1821, doi:10.5194/amt-5-1809-2012, 2012.

$\mathrm{Fu}, \mathrm{Q}$. and Liou, K.: On the correlated k-distribution method for radiative transfer in nonhomogeneous atmospheres, J. Atmos. Sci., 49, 2139-2156, 1992.

Gabella, M., Kisselev, V., and Perona, G.: Retrieval of Aerosol Profile Variations from Reflected Radiation in the Oxygen Absorption A Band, Appl. Opt., 38, 3190-3195, doi:10.1364/AO.38.003190, 1999.

Haring, R., Pollock, R., Sutin, B. M., and Crisp, D.: The Orbiting Carbon Observatory instrument optical design, Current Developments in Lens Design and Optical Engineering V, 2004.

Harrison, R. M. and Yin, J.: Particulate matter in the atmosphere: which particle properties are important for its effects on health?, Sci. Total Environ., 249, 85-101, doi:10.1016/S00489697(99)00513-6, 2000.

Heidinger, A. K. and Stephens, G. L.: Molecular Line Absorption in a Scattering Atmosphere. Part II: Application to Remote Sensing in the O2 A band, J. Atmos. Sci., 57, 1615-1634, doi:10.1175/1520-0469(2000)057<1615:MLAIAS>2.0.CO;2, 2000 .
Hollstein, A. and Fischer, J.: Radiative Transfer Solutions for Coupled Atmosphere Ocean Systems Using the Matrix Operator Technique, J. Quant. Spectr. Radia. Trans., 113, 536-548, 2012.

Hollstein, A. and Lindstrot, R.: Fast reconstruction of hyperspectral radiative transfer simulations by using small spectral subsets: application to the oxygen A band, Atmos. Meas. Tech., 7, 599-607, doi:10.5194/amt-7-599-2014, 2014.

Joiner, J., Yoshida, Y., Vasilkov, A. P., Yoshida, Y., Corp, L. A., and Middleton, E. M.: First observations of global and seasonal terrestrial chlorophyll fluorescence from space, Biogeosciences, 8, 637-651, doi:10.5194/bg-8-637-2011, 2011.

Kokhanovsky, A. A. and Rozanov, V. V.: The determination of dust cloud altitudes from a satellite using hyperspectral measurements in the gaseous absorption band, Int. J. Remote Sens., 31, 2729 2744, 2010.

Koppers, G. A. A., Jansson, J., and Murtagh, D. P.: Aerosol optical thickness retrieval from GOME data in the oxygen A-band, ERS symposium on space at the service of our environment No3, Florence, ITALIE (14/03/1997), 1997.

Lacis, A. A. and Oinas, V.: A description of the correlated k distribution method for modeling nongray gaseous absorption, thermal emission, and multiple scattering in vertically inhomogeneous atmospheres, J. Geophys. Res. Atmos., 96, 9027-9063, 1991.

Levy, R. C., Remer, L. A., and Dubovik, O.: Global aerosol optical properties and application to Moderate Resolution Imaging Spectroradiometer aerosol retrieval over land, J. Geophys. Res. Atmos., 112, D13210, doi:10.1029/2006JD007815, 2007.

Lindstrot, R. and Preusker, R.: On the efficient treatment of temperature profiles for the estimation of atmospheric transmittance under scattering conditions, Atmos. Meas. Tech., 5, 2525-2535, doi:10.5194/amt-5-2525-2012, 2012.

Lohmann, U. and Feichter, J.: Global indirect aerosol effects: a review, Atmos. Chem. Phys., 5, 715-737, doi:10.5194/acp-5-7152005, 2005.

McClain, C. R.: A Decade of Satellite Ocean Color Observations, Ann. Rev. Marine Sci., 1, 19-42, doi:10.1146/annurev.marine.010908.163650, 2009.

McConnell, J. R., Edwards, R., Kok, G. L., Flanner, M. G., Zender, C. S., Saltzman, E. S., Banta, J. R., Pasteris, D. R., Carter, M. M., and Kahl, J. D. W.: 20th-Century Industrial Black Carbon Emissions Altered Arctic Climate Forcing, Science, 317, 1381-1384, doi:10.1126/science.1144856, 2007.

McMichael, A., Campbell-Lendrum, D., Corvalán, C., Ebi, K., Githeko, A., Scheraga, J., and Woodward, A. (Eds.): Climate change and human health, WHO Library Cataloguing-inPublication Data, 2003.

Meijer, Y., Ingmanna, P., Löscher, A., and the CarbonSat Mission Advisory Group Team: CarbonSat: ESA's Earth Explorer 8 Candidate Mission, Geophys. Res. Abstr., 14 EGU2012-2474-1, EGU General Assembly 2012, Vienna, Austria, 2012.

Moré, J. J., Sorenson, D. C., Garbow, B. S., and Hillstrom, K. E.: The MINPACK project, Sources and Development of Mathematical Software, 88-111, 1984.

Muller, J.-P., Preusker, R., Fischer, J., Zuhlke, M., Brockmann, C., and Regner, P.: ALBEDOMAP: MERIS land surface albedo retrieval using data fusion with MODIS BRDF and its validation using contemporaneous EO and in situ data products, Geoscience 
and Remote Sensing Symposium, 2007, IGARSS 2007, IEEE International, 2404 - 2407, 2007.

Natraj, V., Jiang, X., lie Shia, R., Huang, X., Margolis, J. S., and Yung, Y. L.: Application of principal component analysis to high spectral resolution radiative transfer: A case study of the band, J. Quant. Spectr. Radiat. Trans., 95, 539-556, doi:10.1016/j.jqsrt.2004.12.024, 2005.

Nel, A.: Air Pollution-Related Illness: Effects of Particles, Science, 308, 804-806, doi:10.1126/science.1108752, 2005.

Pedregosa, F., Varoquaux, G., Gramfort, A., Michel, V., Thirion, B., Grisel, O., Blondel, M., Prettenhofer, P., Weiss, R., Dubourg, V., Vanderplas, J., Passos, A., Cournapeau, D., Brucher, M., Perrot, M., and Duchesnay, E.: Scikit-learn: Machine Learning in Python, J. Machine Learning Res., 12, 2825-2830, 2011.

Pelletier, B., Frouin, R., and Dubuisson, P.: Retrieval of the aerosol vertical distribution from atmospheric radiance, Proc. SPIE 7150, 71501R, doi:10.1117/12.806527, 2008.

Pöschl, U.: Atmospheric Aerosols: Composition, Transformation, Climate and Health Effects, Angewandte Chemie International Edition, 44, 7520-7540, doi:10.1002/anie.200501122, 2005.

Preusker, R. and Lindstrot, R.: Remote Sensing of Cloud-Top Pressure Using Moderately Resolved Measurements within the Oxygen A Band-A Sensitivity Study, J. Appl. Meteorol. Climatol., 48, 1562-1574, 2009.

Quijano, A. L., Sokolik, I. N., and Toon, O. B.: Influence of the aerosol vertical distribution on the retrievals of aerosol optical depth from satellite radiance measurements, Geophys. Res. Lett., 27, 3457-3460, 2000.

Rascher, U., Gioli, B., and Miglietta, F.: FLEX - Fluorescence Explorer: A Remote Sensing Approach to Quantify SpatioTemporal Variations of Photosynthetic Efficiency from Space, in: Photosynthesis. Energy from the Sun, edited by: Allen, J. F., Gantt, E., Golbeck, J. H., and Osmond, B., 1388-1390, Springer Netherlands, doi:10.1007/978-1-4020-6709-9_299, 2008.

Rodgers, C. D.: Inverse methods for athmospheric sounding, World Scientific Publishing Co. Pte. Ltd., 2000.

Rosen, J. M., Hofmann, D. J., and Laby, J.: Stratospheric Aerosol Measurements II: The Worldwide Distribution, J. Atmos. Sci., 32, 1457-1462, doi:10.1175/15200469(1975)032<1457:SAMITW>2.0.CO;2, 1975.

Rothman, L., Gordon, I., Barbe, A., Benner, D., Bernath, P., Birk, M., Boudon, V., Brown, L., Campargue, A., Champion, J.-P., Chance, K., Coudert, L., Dana, V., Devi, V., Fally, S., Flaud, J.M., Gamache, R., Goldman, A., Jacquemart, D., Kleiner, I., Lacome, N., Lafferty, W., Mandin, J.-Y., Massie, S., Mikhailenko, S., Miller, C., Moazzen-Ahmadi, N., Naumenko, O., Nikitin, A., Orphal, J., Perevalov, V., Perrin, A., Predoi-Cross, A., Rinsland, C., Rotger, M., Simecková, M., Smith, M., Sung, K., Tashkun, S., Tennyson, J., Toth, R., Vandaele, A., and Auwera, J. V.: The HITRAN 2008 molecular spectroscopic database, J. Quant. Spectr. Radiat. Trans., 110, 533-572, doi:10.1016/j.jqsrt.2009.02.013, 2009.

Rozanov, V. V. and Kokhanovsky, A. A.: Semianalytical cloud retrieval algorithm as applied to the cloud top altitude and the cloud geometrical thickness determination from top-of-atmosphere reflectance measurements in the oxygen A band, J. Geophys. Res. Atmos., 109, D05202, doi:10.1029/2003JD004104, 2004.
Sanders, A. F. J. and de Haan, J. F.: Retrieval of aerosol parameters from the oxygen A band in the presence of chlorophyll fluorescence, Atmos. Meas. Tech., 6, 2725-2740, doi:10.5194/amt-62725-2013, 2013.

Sanghavi, S., Martonchik, J. V., Landgraf, J., and Platt, U.: Retrieval of the optical depth and vertical distribution of particulate scatterers in the atmosphere using $\mathrm{O}_{2} \mathrm{~A}$ - and B-band SCIAMACHY observations over Kanpur: a case study, Atmos. Meas. Tech., 5, 1099-1119, doi:10.5194/amt-5-1099-2012, 2012.

Seaton, A., Godden, D., MacNee, W., and Donaldson, K.: Particulate air pollution and acute health effects, The Lancet, 345, 176178, doi:10.1016/S0140-6736(95)90173-6, 1995.

Stoll, M.: The FLEX-Fluorescence Explorer mission project: motivations and present status of preparatory activities, Geoscience and Remote Sensing Symposium, 2003, IGARSS '03, Proceedings, 2003 IEEE International, 1, 585-587, 2003.

Torres, O., Bhartia, P., Herman, J., Ahmad, Z., and Gleason, J.: Derivation of aerosol properties from satellite measurements of backscattered ultraviolet radiation: Theoretical basis, J. Geophys. Res. Atmos., 103, 17099-17110, 1998.

Veefkind, J., Aben, I., McMullan, K., Förster, H., de Vries, J., Otter, G., Claas, J., Eskes, H., de Haan, J., Kleipool, Q., van Weele, M., Hasekamp, O., Hoogeveen, R., Landgraf, J., Snel, R., Tol, P., Ingmann, P., Voors, R., Kruizinga, B., Vink, R., Visser, H., and Levelt, P.: $\{$ TROPOMI $\}$ on the $\{$ ESA $\}$ Sentinel-5 Precursor: A \{GMES\} mission for global observations of the atmospheric composition for climate, air quality and ozone layer applications, Remote Sensi. Environ., 120, 70-83, doi:10.1016/j.rse.2011.09.027, 2012.

Velazco, V. A., Buchwitz, M., Bovensmann, H., Reuter, M., Schneising, O., Heymann, J., Krings, T., Gerilowski, K., and Burrows, J. P.: Towards space based verification of $\mathrm{CO}_{2}$ emissions from strong localized sources: fossil fuel power plant emissions as seen by a CarbonSat constellation, Atmos. Meas. Tech., 4, 2809-2822, doi:10.5194/amt-4-2809-2011, 2011.

West, R., Crisp, D., and Chen, L.: Mapping transformations for broadband atmospheric radiation calculations, J. Quant. Spectr. Radiat. Trans., 43, 191-199, 1990.

Winker, D. M., Vaughan, M. A., Omar, A., Hu, Y., Powell, K. A., Liu, Z., Hunt, W. H., and Young, S. A.: Overview of the CALIPSO Mission and CALIOP Data Processing Algorithms, J. Atmos. Oceanic Technol., 26, 2310-2323, doi:10.1175/2009JTECHA1281.1, 2009.

Winker, D. M., Pelon, J., Coakley, J. A., Ackerman, S. A., Charlson, R. J., Colarco, P. R., Flamant, P., Fu, Q., Hoff, R. M., Kittaka, C., Kubar, T. L., Le Treut, H., McCormick, M. P., Mégie, G., Poole, L., Powell, K., Trepte, C., Vaughan, M. A., and Wielicki, B. A.: The CALIPSO Mission: A Global 3D View of Aerosols and Clouds, Bull. Amer. Meteor. Soc., 91, 1211-1229, doi:10.1175/2010BAMS3009.1, 2010.

Wiscombe, W. J.: Improved Mie scattering algorithms, Appl. Opt., 19, 1505-1509, 1980.

Zieger, P., Ruhtz, T., Preusker, R., and Fischer, J.: Dual-aureole and sun spectrometer system for airborne measurements of aerosol optical properties, Appl. Opt., 46, 8542-8552, 2007. 